\title{
Telescopic observations of Nova Persei.
}

By F. G. Hagen, S. J.

The following observations are a continuation of those published in the Supplementary Notes to the A.S.V., p. 15. These latter were made with the naked eye or a field glass, while the Nova was decreasing from the first to the sixth magnitude. The few remaining observations now published fall within the period of change from magnitude 6.5 to I 1 , They were made with the 12 inch refractor, unless otherwise stated in the Remarks.

The comparison stars are given in Table I. The num. bers under $\mathrm{H}_{2}$ are those of the Second Chart and Catalogue (Suppl. Notes, p. 3), and the corresponding letters refer to the observations in Table III. The steps in the third column differ slightly from the scale of the Circular, as they have been derived from all the observations made. The last column contains in the upper half the photometric magnitudes of the Potsdam Observatory (A. N. 3779), and below three magnitudes from the Harvard College Observatory Circular 66 (Table I).

The corresponding values of steps and magnitudes in Table II were read graphically from a curve constructed by means of Table $I$.

The reduction of the observations in Table III corresponds to that in the Supplementary Notes, p. 13-15, where the necessary explanations about Schönfeld's formulas may be found. The explicit results from both formulas will aid in judging of the accuracy of the single estimates, and will perhaps call attention to the importance of the first formula, which seems to have fallen almost into oblivion.

The magnitudes in the last column before the Remarks were read directly from the plotted curve, but may also be derived from Table II. No correction for the absorption of light has been applied.

Table I. Comparison stars.

\begin{tabular}{r|r|r|r}
\hline $\mathrm{H}_{2}$ & & Steps & Mag. \\
\hline $\mathrm{I}$ & $a$ & 0.0 & 6.57 \\
2 & $b$ & 7.3 & 6.61 \\
3 & $c$ & 17.2 & 7.16 \\
4 & $d$ & 20.5 & 7.50 \\
9 & $n$ & 41.5 & 8.63 \\
$\mathrm{I} 3$ & $s$ & 50.1 & 8.81 \\
$\mathrm{I} 6$ & $p$ & 55.5 & 8.92
\end{tabular}

\begin{tabular}{|c|c|c|c}
\hline $\mathrm{H}_{2}$ & & Steps & Mag. \\
\hline 17 & $q$ & 57.5 & 9.22 \\
19 & $r$ & 60.2 & 9.05 \\
20 & $u$ & 62.0 & 9.11 \\
24 & $x$ & 67.0 & - \\
27 & $w$ & 67.0 & - \\
29 & $y$ & 71.3 & - \\
31 & $\beta$ & 76.6 & -
\end{tabular}

Table II. Scale.

\begin{tabular}{r|c}
\hline Steps & Mag. \\
\hline 0 & 6.42 \\
5 & 6.63 \\
10 & 6.87 \\
15 & 7.12 \\
20 & 7.34
\end{tabular}

\begin{tabular}{c|c}
\hline Steps & Mag. \\
\hline 25 & $7.5^{8}$ \\
30 & 7.81 \\
35 & 8.05 \\
40 & 8.27 \\
45 & 8.49
\end{tabular}

\begin{tabular}{c|c}
\hline Steps & Mag. \\
\hline 50 & 8.71 \\
55 & 8.93 \\
60 & 9.13 \\
65 & 9.33 \\
70 & 9.52
\end{tabular}

\begin{tabular}{c|r}
\hline Steps & Mag. \\
\hline 75 & 9.70 \\
80 & 9.89 \\
85 & 10.09 \\
90 & 10.29 \\
95 & 10.47
\end{tabular}

\begin{tabular}{c|c|c|c}
\hline $\mathrm{H}_{2}$ & & Steps & Mag. \\
\hline 32 & $\alpha$ & 80.6 & 9.71 \\
33 & $\tau$ & 87.9 & - \\
34 & $\mu$ & 92.9 & 10.57 \\
35 & $\pi$ & 96.2 & - \\
40 & $\varphi$ & 106.0 & - \\
$4 \mathrm{I}$ & $\sigma$ & 109.3 & - \\
42 & $A$ & 115.3 & 11.03
\end{tabular}

Table III. The observations.

\begin{tabular}{|c|c|c|c|c|c|c|c|c|c|}
\hline Date & & Gr. M.T. & Sky & Comparisons & $\mathbf{I}$ & II & Mean & Mag. & Remarks \\
\hline \multirow{12}{*}{$\begin{array}{c}\text { I901 June } \\
\text { July }\end{array}$} & 19 & $20^{h} \cdot 1$ & III & $a 6 N_{3} b$ & 5.15 & 4.87 & 5.01 & 6.63 & $N$ more red than $a$ \\
\hline & 24 & 19.2 & II & $N$ го $a$ & 10.00 & - & 10.00 & 6.87 & \\
\hline & I 5 & 17.9 & III & $N \circ a$ & 0.00 & - & 0.00 & 6.42 & $N=a$ in brightness and color \\
\hline & 8 & 17.1 & III & $b 8 N 8 c$ & I 2.25 & 12.50 & $12.3^{8}$ & 6.99 & \\
\hline & 20 & 17.9 & IJ & $b_{3} N_{7} c$ & 10.25 & 10.27 & 10.26 & 6.88 & $N$ white! \\
\hline & 7 & 16.2 & II & ${ }^{b} 6 \mathrm{~N}_{4} \mathrm{c}$ & I 3.25 & 13.24 & 13.24 & 7.02 & $\searrow$ \\
\hline & 8 & 16.0 & - & $b_{4} N 6 c$ & 11.25 & 11.26 & I 1.26 & 6.91 & 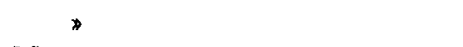 \\
\hline & 21 & 13.7 & II & $b_{7} N_{3} c$ & I 4.23 & 14.23 & 14.23 & 7.09 & Moon \\
\hline & I I & 13.4 & II & $b_{3} N 7 c$ & I 0.25 & 10.27 & 10.26 & 6.91 & 5 -inch telescope \\
\hline & 12 & 15.2 & II & $b 10<2 d 2 N 8 n$ & $(28.00)$ & 24.80 & 25.87 & $7.6 \mathrm{I}$ & \\
\hline & 24 & 13.2 & II & $d_{3} N_{7} n$ & $(29.00)$ & 26.95 & 27.63 & $7 \cdot 7 \mathrm{I}$ & Strong moon \\
\hline & 27 & 14.7 & II & $d \circ N !$ & 20.50 & - & 20.50 & $7 \cdot 37$ & $\rightarrow$ \\
\hline IgO2 Jan. & 5 & 15.9 & I & $d_{3} N 6 n$ & $(29.50)$ & 27.67 & 28.28 & 7.74 & 5 -inch telescope \\
\hline
\end{tabular}




\begin{tabular}{|c|c|c|c|c|c|c|c|c|c|}
\hline & Date & Gr. M.T. & Sky & Comparisons & I & II & Mean & Mag. & Remarks \\
\hline \multirow[t]{8}{*}{1902} & Mar. 20 & I 3 h.o & II & $n_{4} s_{4} p$ I $N_{2} q$ & 56.00 & 56.17 & $5^{6.09}$ & 9.00 & \multirow{8}{*}{$\begin{array}{l}\text { Moon } \\
\text { Strong moon } \\
\text { Near horizon } \\
\text { Strong moon, } \\
\text { near horizon } \\
\text { Strong moon }\end{array}$} \\
\hline & April I 5 & I 4.3 & II & $n 6 s_{3} N 2 p$ & 52.80 & $53 \cdot 34$ & 53.07 & 8.86 & \\
\hline & May I & 13.2 & III & $n_{7} s_{4} N_{2 p}$ & 53.80 & 53.70 & 53.75 & 8.88 & \\
\hline & Aug. I 6 & 17.2 & $\mathrm{II}$ & $p 2 q 3 r_{2} u_{4} w_{2} x$ & & & & & \\
\hline & & & & $x_{5} y_{2} \beta_{4} \alpha 3 N \circ \tau$ & $85 \cdot 75$ & 一 & 85.75 & I O. I I & \\
\hline & Sept. 18 & I 6.9 & II & $\tau_{3} \mu 2 N_{3} \pi$ & 94.05 & 94.22 & 94.14 & 10.46 & \\
\hline & Nov. 28 & 13.9 & II & $\tau 7 \pi 3 N \circ \varphi$ & 102.60 & 一 & 102.60 & I0.73 & \\
\hline & Febr.20 & - & II & $\pi \approx N_{3} \varphi$ & 100.60 & 100.12 & 100.36 & 10.66 & \\
\hline & April 18 & - & I & $\varphi \circ \sigma 3 N 6 A$ & I 10.80 & 111.30 & 111.05 & 11.05 & Near horizon \\
\hline
\end{tabular}

The three numbers in parenthesis were given half weight. The two comparison stars $d$ and $n$ should not have been used together. The interval between them is a full magnitude, and it was in fact underestimated in steps. The step in these three estimates is about twice as large as in the others. This difference will however not affect the results by formula II.

If the number of steps in these and the former observations of the Nova appear larger than what high authorities have recommended, it is well to observe that according to Table II:

Georgetown College Observatory, r 903 Dec. 25. and

$$
\begin{aligned}
& 1 \text { Step }=0.0418 \text { mag. } \\
& 1 \text { Mag. }=240 \text { steps. }
\end{aligned}
$$

Thus the average step here employed is about one half of Schönfeld's step and somewhat less than one half of Argelander's. Bot these observers not unfrequently used half-steps, which taken as the unit measure would make their records similar to those published here. By warning against more than 4 steps they meant to say that an interval estimated in steps should be less than half a magnitude.

\section{G. Hagen, S. J.}

\section{Oppositions-Ephemeride des Planeten (502) [1903 LC].}

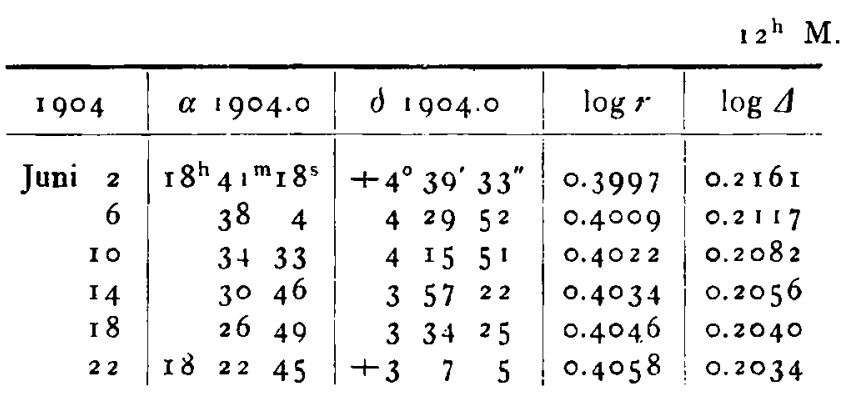

\begin{tabular}{|c|c|c|c|c|c|c|}
\hline \multicolumn{2}{|c|}{1904} & \multicolumn{2}{|c|}{$\alpha 1904.0$} & $d 1904.0$ & \multirow{2}{*}{$\frac{\log r}{0.4070}$} & \multirow{2}{*}{$\frac{\log A}{0.2040}$} \\
\hline Juni & 26 & I $8^{\mathrm{h}}$ & $18^{\mathrm{m}} 39^{\mathrm{s}}$ & $+2^{\circ} 35^{\prime} 36^{\prime \prime}$ & & \\
\hline & 30 & & $14 \quad 35$ & $2 \quad 0 \quad 12$ & 0.4081 & $0.205^{6}$ \\
\hline Juli & 4 & & 1036 & 12116 & 0.4093 & 0.2083 \\
\hline & 8 & & $\begin{array}{ll}6 & 48\end{array}$ & +o 3910 & 0.4104 & 0.2121 \\
\hline & I 2 & & 3 I 3 & $-0 \quad 53^{8}$ & 0.4 I 5 & 0.2169 \\
\hline & I 6 & & 5955 & $-0 \quad 5^{2} 40$ & $0.4 I 26$ & 0.2227 \\
\hline
\end{tabular}

Größe $=14.0$. Opposition Juni 26.

Der Ephemeride, die nach dem Ristenpartschen Vorschlage auf mittlere Örter gestellt ist, liegen meine Elemente A. N. $3^{876}$ zugrunde. Störungen durch Jupiter und Saturn sind berücksichtigt.

Bremen, Dezember 1903 .

Hans Osten.

\begin{tabular}{|c|c|c|c|c|c|c|c|c|c|c|c|c|c|c|}
\hline Planet & 190 & & & $\mathrm{z} . \mathrm{W}$ & Vien & Gr. & & $\alpha$ a & pp. & $\log p . \Delta$ & & $\delta \mathrm{apl}$ & & $\log p . \Delta$ \\
\hline $1904 \mathrm{ND}$ & April & 14 & 9 & $31^{n}$ & $6^{5}$ & $\mathrm{I} 3^{\mathrm{m}} \mathrm{o}$ & $8^{h}$ & $22^{\mathrm{m}}$ & $9^{s} \cdot 94$ & $9.4 ! 4$ & +14 & ${ }^{\circ} 48$ & I 3". 2 & 0.720 \\
\hline $1904 \mathrm{NN}$ & ; & 14 & 9 & 0 & 9 & I 3.5 & 10 & I & 14.44 & 8.700 & +8 & I I & 25.1 & $0.75^{I}$ \\
\hline $1904 \mathrm{NO}$ & 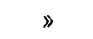 & 3 & 10 & 25 & 12 & - & 10 & I 3 & 7.53 & 9.012 & +13 & 42 & $41 \cdot 4$ & 0.699 \\
\hline 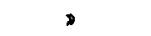 & 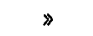 & I 2 & 9 & 0 & 37 & 14.0 & 10 & I I & I & 8.318 & +13 & $5^{2}$ & $21 \cdot 3$ & 0.693 \\
\hline $1904 \mathrm{NQ}$ & $»$ & I 2 & 9 & 47 & 6 & - & I 2 & 28 & 27.22 & $9.12 \mathrm{I}$ & -0 & 11 & 14.6 & $0.8 \times 6$ \\
\hline $1904 \mathrm{NS}$ & 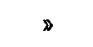 & $\times 3$ & I I & 39 & 47 & - & I 2 & $3^{6}$ & $\mathrm{r}_{4} .87$ & 8.718 & +8 & 54 & 58.7. & 0.745 \\
\hline $1904 \mathrm{NT}$ & $»$ & I 3 & I I & 14 & 54 & - & I 2 & 42 & 7.84 & 7.009 & +9 & 25 & × 2.7 & 0.739 \\
\hline $1904 \mathrm{NV}$ & $\gg$ & $x_{4}$ & I 2 & $3 I$ & 23 & 14.0 & I 3 & 54 & 8.30 & $8.13^{6}$ & $-I$ & 20 & 10.9 & 0.824 \\
\hline
\end{tabular}

Beobachtungen von Planeten auf der k. k. Sternwarte in Wien.

Korrektion der Ephemeriden (Veröff. R. I. 22). (274) Philagoria April 12: - Ios -o:2; (403) Cyane April 12: $-59^{\mathrm{s}}+4: 8 ;(478)$ Tergeste April 13: $-30^{\mathrm{s}}+2 \mathbf{2}^{\prime}$.

Wien, k. k. Sternwarte, I904 April r6.

F. Palisa. 